\title{
OPTIMIZATION OF SIGNAL-TIMING PARAMETERS FOR THE INTERSECTION WITH HOOK TURNS
}

\author{
Yiming Bie ${ }^{1}$, Shaowu Cheng ${ }^{2}$, Zhiyuan Liu ${ }^{3}$ \\ ${ }^{1,2}$ School of Transportation Science and Engineering, Harbin Institute of Technology, China \\ ${ }^{3}$ School of Transportation, Southeast University, China
}

Submitted 31 October 2014; resubmitted 9 March 2015, 24 June 2015; accepted 1 July 2015

\begin{abstract}
A Hook Turn (HT) traffic control scheme has been successfully implemented in urban Melbourne (Australia) ever since 1950s, for the regulation of right-turning vehicles at the intersections (in traffic system where driving is on the left). This paper addresses the optimal signal-timing of the HT scheme, which is still an open question in the literature. Under the HT scheme, right-turning vehicles should enter the intersection and stop at a waiting area. Hence, it is common to have a spillback from these vehicles if the right-turning volume is high. This paper provides an in-depth analysis of the spillback phenomenon on the traffic movements and the average delays, and proposes the models for the calculation of average delay in different cases. With the aim of minimizing the average delay of all the vehicles, a nonlinear integer-programming model is proposed for the optimal signal-timing problem of HT scheme. A Genetic Algorithm (GA) is used to solve this model, considering the complexity of its objective function. A realistic example developed based on one intersection with HT in urban Melbourne is adopted to assess the proposed methodology. Based on real survey data in morning peak and nonpeak hours, we compare the existing signal plan and optimal plan. The numerical test shows that compared with the existing plan, the optimal plan can reduce the average delay for $12.05 \%$ in peak hour and $19.96 \%$ in nonpeak hour. Sensitive analysis is also conducted to investigate the variation of right-turning ratio on the intersection operational performance.
\end{abstract}

Keywords: hook turn; signal-timing; right-turning vehicle; delay model; evaluation.

\section{Introduction}

At the intersections in urban areas, the regulation of right-turning vehicles (in traffic system where driving is on the left) is a significant problem (Yang et al. 2012; Abukauskas et al. 2013). In general, at an intersection with high volume of right-turning vehicles, a dedicated signal phase is used to avoid the conflicts between right-turning vehicles and through vehicles. The dedicated signal phase can improve the safety level and reduce the vehicle delays, yet it requires a dedicated lane for the right-turning vehicles at the intersection (Bugg et al. 2013). Hence, the dedicated right-turning signal phase is not suitable for the intersections with limited space, where right-turning vehicles share one phase and should give the right of way to the through vehicles. The conflicts between right-turning vehicles and through vehicles undermine the safety level, especially if there are trams travelling through the intersection.

Melbourne (Australia) has one of the largest urban tram systems in the world, with a total network distance of over $200 \mathrm{~km}$ (Currie, Smith 2006). The tram rails are located in the middle of the road, which reduces the space for other vehicles in the intersection. Thus, it is difficult to set up a dedicated lane or dedicate signal phase for right-turning vehicles. The right-turning vehicles have to share a lane with the through vehicles, which yields conflicts. In addition, when right-turning vehicles give the right of way to the trams, they block the lane and considerably increase the vehicle delays.

A remedy to the conflicts between trams and right-turning vehicles was proposed in Melbourne in the 1950s, named as Hook Turn (HT) scheme, and HT has been implemented in tens of intersections in urban Melbourne ever since then (Currie, Smith 2006). Fig. 1a shows an intersection with HT manoeuvre (for rightturning vehicles at the North/South leg), and Fig. 1b shows a conventional intersection.

We can see from Fig. 1a that the right-turning lane (a shared lane with through and left-turning vehicles) is relocated from the far side to the curbside. The HT scheme requires the right-turning vehicles at South/North leg enter the intersection during the green time, and then stop at and wait in the waiting area.

Corresponding author: Zhiyuan Liu

E-mail: jszodiac@gmail.com 

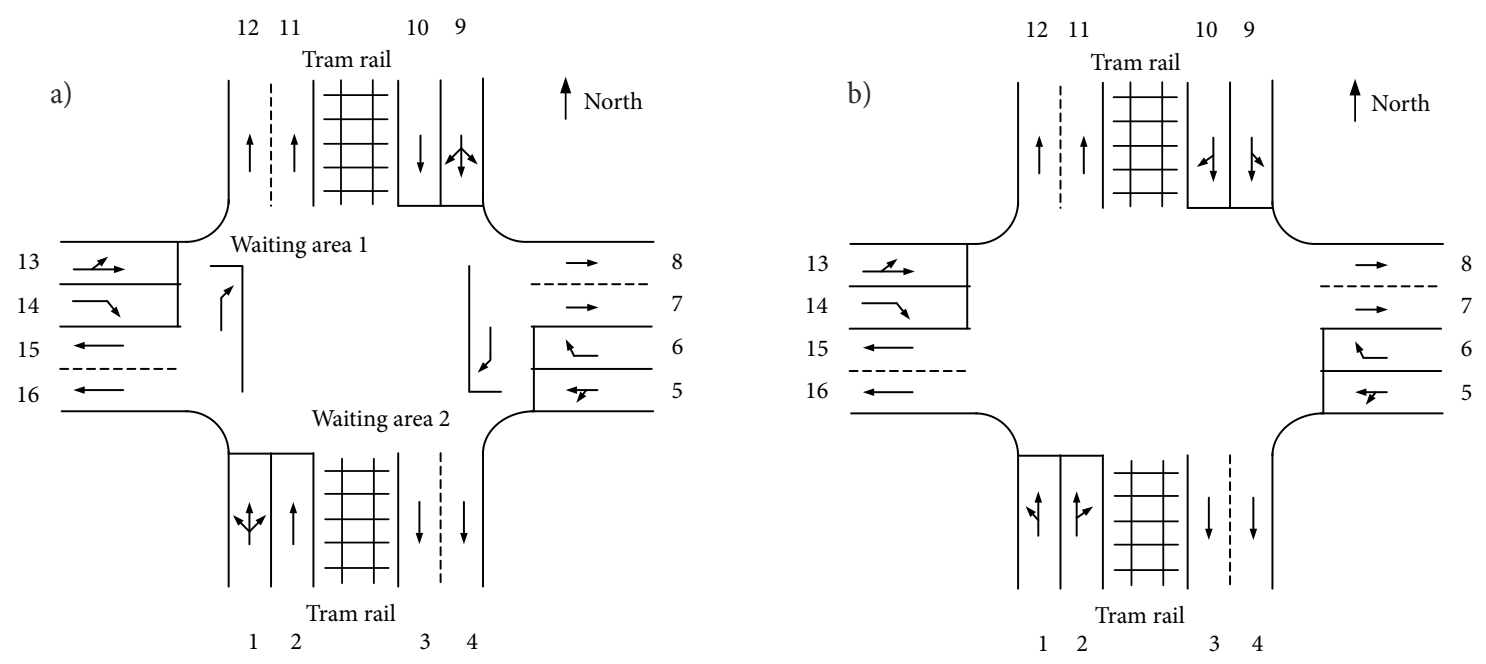

Fig. 1. Comparison of a hook-turn intersection and traditional intersection: $\mathrm{a}$ - intersection with HT; $\mathrm{b}$ - traditional intersection

After the signal light of the side road turns green, the vehicles in the waiting area should then depart and leave the intersection, followed by the vehicles on the side road (lanes 5, 6, 13 and 14). The track of these vehicles in the intersection looks like a hook, thus it is named as a HT. For the sake of presentation, these vehicles are called as HT vehicles.

Comparing Fig. 1a with Fig. 1b, we can see that the HT scheme has two unique features: (1) the HT vehicles are relocated to the curbside; (2) the HT vehicles should stop twice, once at the stop line and once in the waiting area.

Although HT scheme has been smoothly implemented in Melbourne and several other cities for tens of years (Currie, Reynolds 2011), the theoretical studies are quite scarce. Andrew O'Brien and Associates (2000) conducted a review of HTs to determine whether the use of HTs should be continued, expanded, modified or reduced. The safety and operational effects of these turns in Melbourne city were evaluated. The study did not find any evidence to suggest that there was anything inherently 'bad' about HTs. The investigations suggested that the HTs served central Melbourne well, particularly in relation to reducing tram delays and keeping pedestrian crash number low, while still retaining maximum mobility.

Currie and Reynolds (2011) also conducted a review of the HT and explored operations and safety impacts. Operational analysis of the traffic impacts of HTs in Melbourne suggested that they acted to reduce congestion because turning traffic did not delay through vehicles. In addition, $38 \%$ of drivers tended to avoid HTs and each tram delay was decreased from 11.25 to 15.64 seconds. A series of safety analyses with crash data and conflict point analysis demonstrated that intersections with HTs had better safety performance than conventional intersections.

Hounsell and Yap (2013) compared the traffic performance of a HT intersection with an equivalent conventional intersection with opposed right turns based on simulations. It was found that HTs reduced delays to through traffic from the same approach in nearly all cases. Overall intersection performance depended on the scale of any increased delays to left-turning traffic and side road traffic resulting from the HT movement. Pai et al. (2013) studied the motorcycles' violating behaviours at the intersections with Hook Turn Area (HTA) in Taiwan. However, the HT area of the intersection is for motorcyclists, not for automobile. They found that there was an increased likelihood of HTA-violation at $\mathrm{T} / \mathrm{Y}$ intersections during non-rush hours in rural areas.

The existing literature mainly investigates the efficiency and safety issues of HT scheme. How to design the optimal signal-timing plan based on real traffic flow data is still an open question. A suboptimal signal-timing plan has inherent impacts on the vehicle movements and service level of the intersection. Fig. 1 shows that the HT scheme is quite different with traditional signal plan, thus the existing signal optimization method for the traditional plan is not suitable for the HT case. Moreover, many of the implemented HT plans in Melbourne adopt fixed-time signal plan, which are not the optimal plan subject to the traffic flows. Therefore, it is a timely issue with practical significance to cope with the optimal signal-timing, which is taken as the focus of this study.

This study focuses on a signalized intersection with HT scheme. Based on an in-depth analysis of the traffic flow properties, this paper first proposes the models between average vehicle delay and traffic volume of each stream. Due to the limited space of the waiting area in the intersection, it is common to have a spillback from the HT vehicles. The impacts of such a spillback on the traffic movements are fully discussed. Then, with the objective of minimizing the average delay of all the vehicles, a nonlinear integer-programming model is proposed for the optimal signal plan. Due to the complexity of the objective function, it is difficult to calculate its gradient, thus a Genetic Algorithm (GA) is used to solve the proposed model. This paper thus fills the gaps of optimal signal-timing algorithm for HT scheme, and 
the resultant optimal plan can significantly reduce the average vehicle delay at the intersection.

The remainder of this paper is organized as follows. Section 1 presents the models for the average delay of left-turning, right-turning and through movements at an intersection with HT scheme, and then proposes the optimization model for the signal-timing problem. In Section 2, a realistic example is developed based on an intersection in urban Melbourne to assess the proposed methodology. The performance of the optimal plan and the current plan is holistically contrasted. Finally, last section concludes this paper.

\section{Development of the Signal-Timing Algorithm}

The objective for the optimal signal-timing is to minimize the total average delay of the intersection. In this section, the vehicle delay models for different traffic flows are developed firstly, and then the total average delay model is obtained where signal-timing parameters are expressed as variables.

The setting of signal phase is the base of signal control. For instance, the signal phases of the intersection shown in Fig. 1a are given by Fig. 2. The South-bound and North-bound flows are controlled by phase 1 . There is no hook-turn vehicle in East and West approaches and a dedicated phase (phase 3 ) is set for the right-turning vehicles.

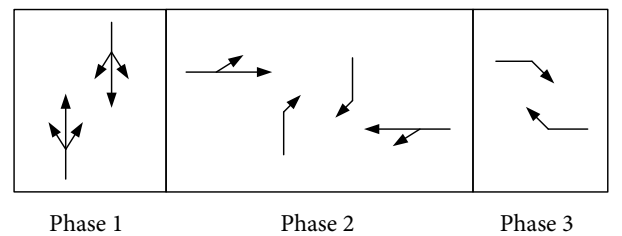

Fig. 2. The phasing diagram for the intersection with HTs

\subsection{Measurement of the Vehicle Delay}

Taking Fig. 1 as an example, compared with traditional intersection, the unique features of vehicle movements at HT intersection include:

- the HT vehicles should wait at the waiting area of the intersection;

- after phase 2 turns green, the vehicles at lanes 5 and 13 need to wait until the HT vehicles at the waiting area leave the intersection, thus their effective green time is diminished;

- the through driving vehicles at lanes 1, 2, 9 and 10 have no conflict with the right-turning vehicles and can travel smoothly during green time;

- due to the limited space of waiting area in the intersection, it can only accommodate few HT vehicles, thus when the volume of HT vehicles is high, a spillback will commonly occur.

Such a spillback will block the movement of leftturning vehicles on the same lane, and drastically increase the delay of left-turning vehicles.

We assume that the vehicle arrival follows a Poisson distribution, and the cycle length of intersection is
$C$ seconds. Let $N$ denote the number of signal phases. The green time of phase $i$ is denoted by $g_{i}$ and its intergreen time is $I_{i}$. Let $S_{j}$ denote the saturation flow rate of approaching lane $j$. In the following sub-section, we still take the intersection in Fig. 1a as an example, to elaborate the measurement of vehicle delays.

\subsubsection{Measurement of Vehicle Delay of Phase 1}

Vehicle delay of dedicated through lanes. In Fig. 1, the through driving vehicles at lanes 2 and 10 are not affected by the HT vehicles. Considering that the intersection could be oversaturated, the average delay is calculated by using the following delay function in Highway Capacity Manual (2000):

$$
\begin{aligned}
& \bar{d}_{1 s}=\frac{0.5 \cdot C \cdot\left(1-\lambda_{1}\right)^{2}}{1-\left(\min \left(1, x_{1 s}\right) \cdot \lambda_{1}\right)}+ \\
& 900 \cdot T \cdot\left(\left(x_{1 s}-1\right)+\sqrt{\left(x_{1 s}-1\right)^{2}+\frac{8 \cdot K x_{1 s}}{C a p_{1 s} \cdot T}}\right),
\end{aligned}
$$

where: $\bar{d}_{1 s}$ is the average vehicle delay of a through lane under phase 1 [seconds]; $\lambda_{1}$ is the green split; $x_{1 s}$ is the saturation degree of a dedicated through lane under phase 1; $\operatorname{Cap}_{1 s}$ is the vehicle capacity of the lane $[\mathrm{pcu} / \mathrm{h}] ; T$ is the length of study period, whose default value is $0.25 \mathrm{~h} ; K$ is an adjustment parameter, with a default value of 0.5 .

Vehicle delay of shared lanes. We can see that on approaching lanes 1 and 9, the left-turning, through and right-turning vehicles share the lane. In the green time of phase 1, HT vehicles enter the waiting area and wait there. When there is no spillback from the HT vehicles, all the vehicles on these approaching lanes can move smoothly, and their delay before passing the stop line can be calculated by Eq. (1). However, if the spillback occurs, then the vehicle movements on the approaching lane will be blocked, which drastically increases the vehicles delay.

The delay of HT vehicles consists of two components: the delay before passing the stop line at lanes 1 or 9 and the delay at the waiting areas 1 or 2 . In this subsection, we only calculate the first component that is the delay during phase 1 , while the second component will be discussed in subsection 1.1.2 below.

We use $j$ to denote the shared approaching lane (in Fig. $1 j$ can be 1 or 9), and the total volume of lane $j$ is $q_{1 j}$. The volumes of through, left-turning and rightturning vehicles are $q_{1 j_{-} t}, q_{1 j_{-} l}$ and $q_{1 j_{-} r}$, respectively. If the shared lane only allows left-turn and right-turn (through vehicles are forbidden), then $q_{1 j_{\_} t}=0$. Let $k$ denote the waiting area ( $k$ takes 1 or 2 in Fig. 1), and $Q_{k}$ be the maximum number of vehicles that can be accommodated by area $k$. In the green time of phase 1 (denoted by $g_{1}$ ), if the number of HT vehicles passing the stop line (denoted by $Q_{1 j_{r} r}$ ) is higher than $Q_{k}$, the spillback from area $k$ will take place. Otherwise, there will be no spillback. Apparently, the spillback phenomenon is affected by the length of green time $g_{1}$. 
Let $S_{j}$ denote the saturation flow rate of lane $j$, and $Q_{1 j}^{s}$ the maximum number of vehicles that can be released during phase 1 . We have that:

$$
Q_{1 j}^{s}=\frac{g_{1} \cdot S_{j}}{3600} .
$$

Considering the ratio of right-turning vehicles, the maximum number of right-turning vehicles that can be released (denoted by $Q_{1 j r}^{s}$ ) is:

$$
Q_{1 j r}^{s}=Q_{1 j}^{s} \cdot\left(\frac{q_{1 j_{-} r}}{q_{1 j}}\right) .
$$

Here, we know that: (1) if $Q_{1 j r}^{s} \leq Q_{k}$, there will be no spillover, and the lane $j$ can be operated smoothly. Hence, the average delay can be calculated by following Eq. (1); if $Q_{1 j r}^{s}>Q_{k}$, the spillback could happen, and the average delay is specified and calculated according to the following two cases.

Case 1. If the spillback really occurs.

Following the definition of Poisson distribution, during one signal cycle time ( $c$ seconds), the probability of right-turning vehicles are more than $Q_{k}$ is:

$$
P\left(Q_{1 j_{-} r}>Q_{k}\right)=1-P\left(Q_{1 j_{-} r} \leq Q_{k}\right) \text {. }
$$

Due to the limited space of the intersection, the waiting area can only accommodate limited number of HT vehicles (e.g., in the intersections at urban Melbourne, $Q_{k}$ is usually $3 \mathrm{pcu}$ ). Then, we can see that:

$$
\begin{aligned}
& P\left(Q_{1 j_{-} r} \leq Q_{k}\right)=\sum_{A=1}^{Q_{k}} P(A), \\
& A=0,1,2, \ldots, Q_{k},
\end{aligned}
$$

where: $P(A)$ is the probability of $A$ arrivals in one cycle ( $c$ seconds) and it equals:

$$
P(A)=\frac{\left(q_{1 j_{-} r} C\right)^{A} \cdot e^{-q_{1 j_{-} r} C}}{A !} .
$$

When there is a spillback, the number of HT vehicles passing the stop line is $Q_{k}+1$. As per the ratio of HT volume, we can infer that the total number of vehicles passing the stop line (denoted by $Q_{1 j}^{s o}$ ) is:

$$
Q_{1 j}^{s o}=\frac{\left(Q_{k}+1\right) \cdot q_{1 j}}{q_{1 j_{-} r}} .
$$

So far, the elapsed green time of phase 1 is $g_{1}^{s o}$, and we have:

$$
g_{1}^{s o}=q_{1 j} \cdot \frac{C-g_{1}}{S_{j}-q_{1 j}} .
$$

The remaining green time of phase 1 is $g_{1}-g_{1}^{s o}$. However, the existing vehicles on lane $j$ are not able to pass the stop line and get through the intersection, due to the spillback. It implies that the effective green time of lane $j$ is $g_{1}^{s o}$, and its red time increases for $g_{1}-g_{1}^{s o}$ seconds. Consequently, based on Eq. (1), the average delay of vehicles on the shared lane, denoted by $\bar{d}_{1 h}^{\prime}$, can be measured by:

$$
\begin{aligned}
& \bar{d}_{1 h}^{\prime}=P\left(Q_{1 j_{-} r}>Q_{k}\right) \times \\
& \left(\frac{0.5 \cdot C \cdot\left(1-\lambda_{1}^{s o}\right)^{2}}{1-\left(\min \left(1, x_{1 j}\right) \lambda_{1}^{s o}\right)}+\right. \\
& \left.900 \cdot T \cdot\left(\left(x_{1 s}-1\right)+\sqrt{\left(x_{1 j}-1\right)^{2}+\frac{8 \cdot K x_{1 j}}{C a p_{1 j} \cdot T}}\right)\right),
\end{aligned}
$$

where: $\lambda_{1}^{s o}=\frac{g_{1}^{s o}}{C}$ is the actual green split. $x_{1 j}$ is the saturation degree of the shared lane $j . C_{a} p_{1 j}$ is the vehicles capacity of lane $j$ (now equals $S_{j} \cdot \lambda_{1}^{s o}$ ) [pcu/h].

Case 2. When there is no spillback.

In Eq. (5), $P\left(Q_{1 j_{-} r} \leq Q_{k}\right)$ is the probability of not having a spillback. In such case, the average delay can still be measured by using Eq. (1). Hence, in case 2, when there is no spillback, the average vehicle delay, denoted by $\bar{d}_{1 h}^{\prime \prime}$, is measured by:

$$
\begin{aligned}
& \bar{d}_{1 h}^{\prime \prime}=P\left(Q_{1 j_{-} r} \leq Q_{k}\right) \times \\
& \left(\frac{0.5 \cdot C \cdot\left(1-\lambda_{1}\right)^{2}}{1-\left(\min \left(1, x_{1 j}\right) \lambda_{1}\right)}+\right. \\
& \left.900 \cdot T \cdot\left(\left(x_{1 j}-1\right)+\sqrt{\left(x_{1 j}-1\right)^{2}+\frac{8 \cdot K x_{1 j}}{C a p_{1 j} \cdot T}}\right)\right) .
\end{aligned}
$$

Combing the above two cases, the average vehicle delay when $Q_{1 j r}^{s}>Q_{k}$ can be taken as:

$$
\bar{d}_{1 h}=\bar{d}_{1 h}^{\prime}+\bar{d}_{1 h}^{\prime \prime} \text {. }
$$

Taking Fig. 1a as an example, the total delay $d_{1}$ of all the vehicles during one signal cycle equals:

$$
\begin{aligned}
& d_{1}=\frac{C}{3600} \cdot\left(\bar{d}_{1 s_{-} 2} \cdot q_{1 s_{-} 2}+\bar{d}_{1 s_{-} 10} \cdot q_{1 s_{-} 10}+\right. \\
& \left.\bar{d}_{1 h_{-} 1} \cdot q_{1 h_{-} 1}+\bar{d}_{1 h_{-} 9} \cdot q_{1 h_{-} 9}\right),
\end{aligned}
$$

where: $q_{1 s_{-} 2}$ is the arrival volume of through vehicles on lane 2 under phase $1, q_{1 h_{1}}$ is the vehicle volume of shared lane $1, \bar{d}_{1 h_{-} 1}$ and $\bar{d}_{1 s_{-} 2}$ is the average vehicle delay of lane 1 and lane 2 , respectively.

\subsubsection{Measurement of Vehicle Delay of Phase 2}

We can see in Fig. 1a that the HT vehicles enter the intersection during phase 1 , and then they will only leave the intersection during phase 2 . The delay of HT vehicles in the waiting area is thus also affected by phase 2. During phase 2 the number of HT vehicles in the waiting area $k$, denoted by $Q_{2 w k}$, can be measured by:

$$
Q_{2 w k}=\min \left(\frac{C \cdot q_{1 j_{-} r}}{3600}, Q_{1 j r}^{s}\right) .
$$

The delay of HT vehicles in the waiting area, denoted by $\bar{d}_{2 \_w}$, can be calculated by:

$$
\bar{d}_{2_{-} w}=\left(0.5 \cdot g_{1}+I_{1}\right)+\frac{0.5 \cdot Q_{2 w k} \cdot 3600}{S_{2 w k}},
$$

where: $g_{1}$ is the green time of phase $1 ; I_{1}$ is the intergreen 
time of phase $1 ; S_{2 w k}$ is the saturation flow of the rightturning vehicles in the waiting area.

Phase 2 controls the through vehicles of East/West leg and right-turning vehicles in the waiting areas. The vehicles at lanes 5 and 13 can only start to move after the HT vehicles in the waiting areas are released, which diminishes the effective green time of through and rightturning vehicles at lanes 5 and 13 . Let $t_{2 w k}$ denote the duration over which HT vehicles are release from waiting area $k$, and it can be calculated by:

$$
t_{2 w k}=\frac{Q_{2 w k} \cdot 3600}{S_{2 w k}} \text {. }
$$

Let $g_{2 e}$ be the effective green time for vehicles on lanes 5 and 13, and it equals:

$$
g_{2 e}=g_{2}-t_{2 w k} \text {. }
$$

The average delay of vehicles on lanes 5 (13) is denoted by $\bar{d}_{2-5}\left(\bar{d}_{2 \_13}\right)$, and it can be calculated by following Eq. (1), while replacing the green time ratio by $\frac{g_{2 e}}{C}$. Therefore, the overall delay of vehicles in phase 2 , denoted by $d_{2}$, is given by:

$$
d_{2}=\frac{C}{3600} \cdot\left(\bar{d}_{2 \_5} \cdot q_{2 \_5}+\bar{d}_{2 \_13} \cdot q_{2 \_13}\right),
$$

where: $q_{2}{ }_{5}$ is the arrival volume of approaching lane 5; $q_{213}$ is the arrival volume of approaching lane 13 .

\subsubsection{Measurement of Vehicle Delay of Phase 3}

Under phase 3, the movements of vehicles on lanes 6 and 14 are the same with a traditional intersection (Chen et al. 2013). Hence, the delay of these vehicles can be calculated by using Eq. (1). The overall delay of all the vehicles under phase 3 can also be similarly measured by Eq. (12).

\subsection{Optimization Model for the Signal-Timing Plan}

With the vehicle delay of each phase measured above, we can now calculate the average vehicles delay across the entire signal cycle, denoted by $\bar{d}$ :

$$
\bar{d}=\frac{d_{1}+d_{2}+d_{3}}{Q},
$$

where: $Q$ is the number of the total arrivals in one signal cycle.

With the objective of minimizing the average delay, an optimization model is proposed as follows for the optimal signal-timing plan:

$$
\begin{aligned}
& \min \bar{d}=\text { function }\left(C, g_{i}\right) \\
& \text { subject to: } \\
& \sum_{i=1}^{N}\left(g_{i}+I_{i}\right)=C ; 1^{*} \text { MERGEFORMAT } \\
& C_{\min } \leq C \leq C_{\max } ; \\
& g_{i \min } \leq g_{i} \leq g_{i \max } .
\end{aligned}
$$

Note that the average delay rather than total delay is adopted as the objective. This is because the signal cycle length would be adjusted during the optimization and thus affects the number of arrival vehicles in one cycle. The total delay is not only affected by the signal-timing plan but also the number of arrival vehicles. Hence, the total delay cannot reflect the relationship between signal-timing plan and the traffic flow performance.

The decision variables of the model Eqs (19)-(22) include the cycle length $C$ and the green time $g_{i}$ of phase $i$, which all take nonnegative integer values. Therefore, this model is an integer program. We rewrite the model as follows, by substituting the term $C$ based on Eq. (20), which gives:

$$
\min \bar{d}=\text { function }\left(g_{i}\right)
$$

subject to:

$$
\begin{aligned}
& g_{i \min }-g_{i} \leq 0 ; \\
& g_{i}-g_{i \max } \leq 0 .
\end{aligned}
$$

Eqs (23)-(25) define the objective function. We can see that this objective function is highly nonlinear, nonconvex and also a stepwise function. The green time $g_{i}$ takes integer values, thus model Eq. (23) is a mixed nonlinear integer program (Wang et al. 2013; Liu et al. 2014). Hence, it is a NP (Non-deterministic Polynomialtime)-hard problem, which is difficult and inefficient to be solved by any gradient-based algorithms in the literature.

For such sort of complex engineering-based problem, some existing heuristics are more suitable to be taken as the solution method, which does not require the calculation of gradient. In this section, we adopt the GA. GA consists of three main processes: selection, crossover and mutation. For the addressed problem, each chromosome defines a signal green time plan $g_{i}$, which can be conveniently used to calculate the value of objective function and the Selection process. The initial generation of chromosomes as well as crossover and mutation are all conducted with the aids of pseudo random numbers. Due to the space limit, the details of GA are not further included here. Any interested readers are referred to the papers - Park et al. (1999), Ceylan and Bell (2004), Teklu et al. (2007), Liu et al. (2013).

\section{Numerical Experiments}

\subsection{Experimental Data Collection}

A numerical example is used in this section to verify the proposed methodology. As shown in Fig. 3, this example is developed based on the HT intersection in Melbourne city, between the Elizabeth Street and La Trobe Street. These two streets both have bi-directional tramlines. A field traffic survey at this intersection is conducted in the morning peak (8:15-8:45 am) and morning off-peak periods (9:30-10:00 am) on 12 June 2014. The data collected include intersection geometry, channelization, the traffic volumes, saturation flow rates, and volume ratio of each stream, the signal cycle length, green times, intergreen times, etc. The traffic volumes are tabulated in Table 1. 


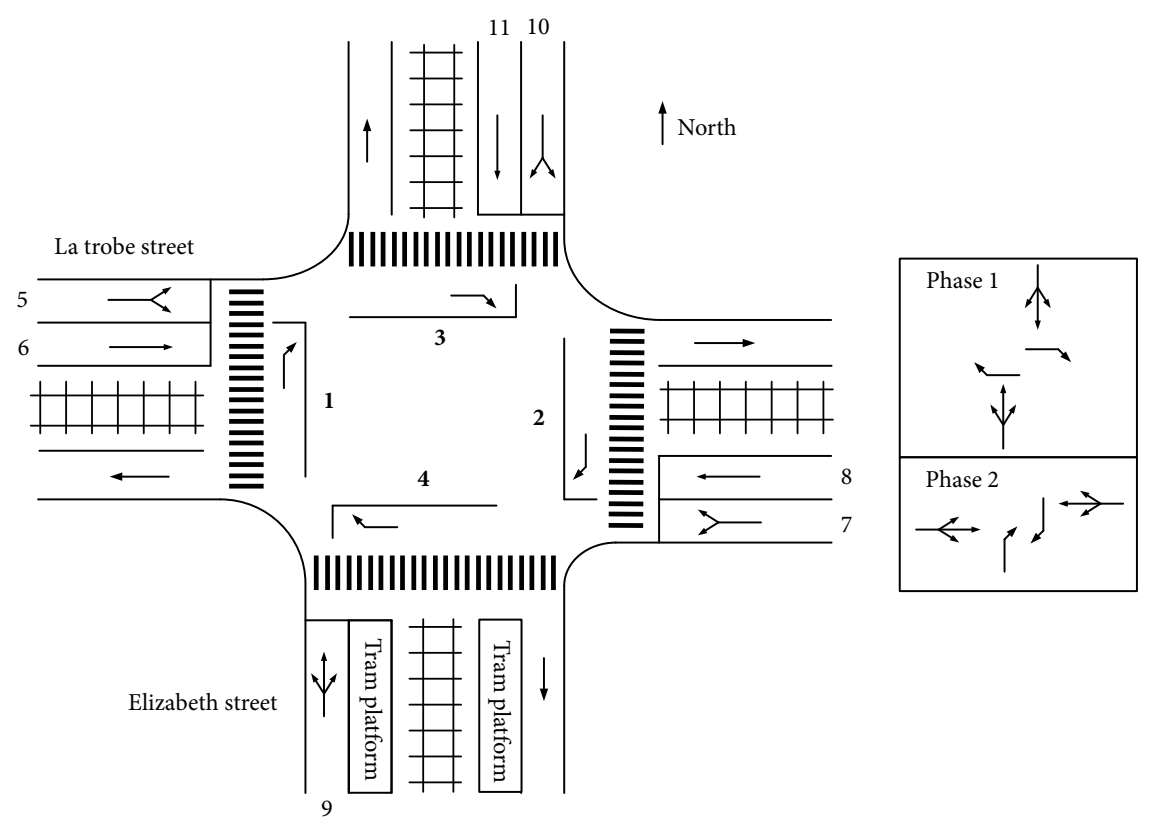

Fig. 3. The channelization of Elizabeth Street and La Trobe Street intersection

Table 1. The traffic volumes obtained from field experiment [pcu/h]

\begin{tabular}{|l|c|c|c|c|c|c|}
\hline \multirow{2}{*}{ Leg } & \multicolumn{3}{|c|}{ Morning peak hour } & \multicolumn{3}{c|}{ Off-peak hour } \\
\cline { 2 - 8 } & Left-turn & Through & Right-turn & Left-turn & Through & Right-turn \\
\hline South leg & 126 & 274 & 96 & 112 & 228 & 68 \\
\hline North leg & 208 & 416 & 138 & 168 & 340 & 114 \\
\hline East leg & 246 & 442 & 120 & 204 & 368 & 76 \\
\hline West leg & 212 & 436 & 112 & 176 & 356 & 64 \\
\hline
\end{tabular}

From Fig. 3, we can see that, to avoid the conflicts between right-turning vehicles and trams, the HT manoeuvre is adopted at all the four legs. Hence, there are four waiting areas in the intersection. This intersection has two signal phases. During the survey period, it executes fixed signal-timing plan (not adaptive control plan), with cycle length of 90 seconds. The green time of phase 1 is 38 seconds, and the green time of phase 2 is 40 seconds. The intergreen times of these two phases are both 6 seconds.

In Fig. 3, each of the four waiting area can only accommodate three cars. The spillback phenomenon of HT vehicles is relatively common during the peak hours, compared with the nonpeak hours. In view of the relatively low volume of trams, we focus only on the average delay of automobile vehicles excluding the trams. However, if the objective of signal-timing is to minimize the average delay of the passengers (rather than the vehicles), the tram delays should be accounted for, because there are more passengers in the trams.

\subsection{Output of Optimal Signal-Timing Plan}

The traffic survey reveals that at intersections with actuated signal control schemes in urban Melbourne, the maximum green time is 50 seconds and the minimum green time is 15 seconds. Therefore, the maximum and minimum green time of this example are set as 50 and 15 seconds respectively. Based on the analysis of the video records, the saturation flow of right-turning vehicles in the waiting area is $1200 \mathrm{pcu} / \mathrm{h}$, and the saturation flow of all the other approaching lanes is $1520 \mathrm{pcu} / \mathrm{h}$.

The GA is then used to solve the optimal signaltiming plan of this example, where the cap of generations is 200 and population size of each generation is 50. The crossover rate is 0.8 and the mutation rate is 0.05. The program is coded in MATLAB (http://www. mathworks.com) to solve the optimal timing plan. Consequently, in the peak hour of 8:15-8:45 am, the optimal signal-timing plan is: $C=88 \mathrm{~s}, g_{1}=39 \mathrm{~s}, g_{2}=37 \mathrm{~s}$. While, in the nonpeak hour of 9:30-10:00 am, the optimal plan is $C=69 \mathrm{~s}, g_{1}=30 \mathrm{~s}, g_{2}=27 \mathrm{~s}$.

\subsection{Comparison and Analysis of the Vehicle Delays}

In order to fully assess the existing signal-timing plan and the optimal plan obtained in Section 2.2, the following four simulation scenarios have been established in VISSIM (http://vision-traffic.ptvgroup.com) for the intersection in Fig. 3.

- A1 - morning nonpeak, existing signal plan;

- A2 - morning nonpeak, optimal signal plan;

- B1 - morning peak, existing signal plan;

- B2 - morning peak, optimal signal plan. 
In the simulation, each scenario has been operated for 10 times, and different random seed are used each time. Table 2 provides the vehicle average delay of each scenario.

With a significance level of $\alpha=0.05$, we perform hypothesis test ( $t$-tests) for the two A scenarios and two $\mathrm{B}$ scenarios, respectively, to test whether there is a significant difference between scenario $\mathrm{A} 1$ and $\mathrm{A} 2$, as well as between B1 and B2. The null hypothesis $H_{0}$ is assuming the mean value of scenario A1 (B1) equals that of A2 (B2). The statistical results are given in Table 3, where $\bar{D}_{\mathrm{A} 1}$ is the average delay of scenario $\mathrm{A} 1, t_{\text {sta }}$ is the value of the test statistic, and $t_{1-\alpha / 2}$ is the critical value.

From Table 3, we can see that in the two tests, the $H_{0}$ hypothesis is rejected, implying that the difference between the existing plan and optimal plan is significant. The data in Table 2 shows that the delay of the optimal plan is lower than that of the existing plan in almost all the tests. Furthermore, the mean value of scenario A1 is 55.11 seconds, while the mean value of A2 is 48.47 seconds that is $12.05 \%$ lower. The mean value of B2 is $19.96 \%$ lower than that of B1.

Table 2. Simulation results of the four scenarios

\begin{tabular}{|c|c|c|c|c|}
\hline \multirow{2}{*}{$\begin{array}{c}\text { Random } \\
\text { seed }\end{array}$} & \multicolumn{4}{|c|}{ Average vehicle delay } \\
\cline { 2 - 5 } & $\mathrm{A} 1$ & $\mathrm{~A} 2$ & $\mathrm{~B} 1$ & $\mathrm{~B} 2$ \\
\hline 10 & 58.67 & 51.88 & 47.43 & 38.20 \\
\hline 20 & 55.38 & 50.34 & 53.09 & 36.36 \\
\hline 30 & 52.11 & 45.81 & 53.04 & 37.01 \\
\hline 40 & 52.19 & 53.40 & 48.57 & 41.39 \\
\hline 50 & 53.06 & 42.41 & 50.81 & 39.68 \\
\hline 60 & 57.73 & 47.26 & 44.91 & 38.22 \\
\hline 70 & 53.03 & 46.58 & 44.65 & 43.97 \\
\hline 80 & 57.51 & 51.19 & 50.37 & 41.02 \\
\hline 90 & 52.95 & 51.54 & 53.35 & 40.60 \\
\hline 100 & 58.43 & 44.24 & 55.21 & 45.01 \\
\hline Mean & 55.11 & 48.47 & 50.14 & 40.15 \\
\hline
\end{tabular}

Table 3. $t$-test results of the four scenarios

\begin{tabular}{|c|c|c|c|c|}
\hline Scenario & $H_{0}$ & $\mathrm{t}_{\text {sta }}$ & $t_{1-\alpha / 2}$ & Result \\
\hline A1 \& A2 & $\bar{D}_{\mathrm{A} 1}=\bar{D}_{\mathrm{A} 2}$ & 4.70 & 2.26 & rejection \\
\hline $\mathrm{B} 1$ \& B2 & $\bar{D}_{\mathrm{B} 1}=\bar{D}_{\mathrm{B} 2}$ & 6.75 & 2.26 & rejection \\
\hline
\end{tabular}

\subsection{Sensitive Analyses}

Due to the limited space of waiting area in the intersection, it can only accommodate several HT vehicles. Thus, a spillback would take place when the volume of HT vehicles is high, which leads to the increase of HT vehicle delay and average intersection vehicle delay. In this subsection, the impacts of the variation of HT vehicle ratio on the intersection operational performances are analysed, while keeping the total traffic demand as a constant.
The intersection shown in Fig. 3 is taken as an example. In Table 1, the total traffic volumes of East/ West leg are $808 \mathrm{pcu} / \mathrm{h}$ and $706 \mathrm{pcu} / \mathrm{h}$ respectively. Four scenarios are designed by changing the ratios of rightturning volume to through volume in East/West leg, while keeping other ratios and traffic demand the same as Table 1.

- C1 - in East/West leg the left-turning, right-turning and through vehicle ratios are 25, 10 and 65\% respectively.

- C2 -in East/West leg the left-turning, right-turning and through vehicle ratios are 25, 20 and 55\% respectively.

- C3 -in East/West leg the left-turning, right-turning and through vehicle ratios are 25, 30 and $45 \%$ respectively.

- C4 -in East/West leg the left-turning, right-turning and through vehicle ratios are 25, 40 and 35\% respectively.

We can see that the left-turning ratio does not change at all. Yet, the righting-turning ratio increases from 10 to $40 \%$, while the through ratio decreases from 65 to $35 \%$.

The optimal signal-timing plans for the four scenarios are obtained firstly. Then VISSIM simulations are conducted to collect the following three evaluation indexes (Fig. 4):

- E1 - average delay of right-turning vehicles in East/West leg.

- E2 - average delay of through vehicles in East/ West leg.

- E3 -average delay of all the vehicles crossing the intersection.

The timing plans and values of evaluation indexes are shown in Table 4.

In Table 1, the right-turning vehicle ratios in East/ West leg are about $15 \%$. From Table 4 we can see that the difference in optimal timing plans between subsection 2.2 and scenarios $\mathrm{C} 1$ and $\mathrm{C} 2$ is not significant. This is because the right-turning ratios under scenarios $\mathrm{C} 1$ and C2 are 10 and $20 \%$. Both of them are close to $15 \%$. In addition, the number of arrival right-turning vehicles at each cycle is not large. Thus, there is nearly no spillover in waiting areas.

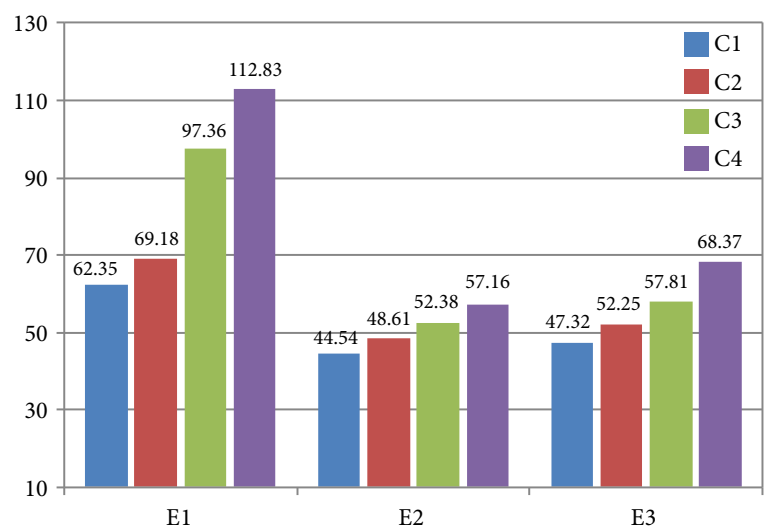

Fig. 4. Comparisons of three evaluation indexes in different scenarios 
However, the optimal cycle lengths under scenarios C3 and C4 decrease to $77 \mathrm{~s}$ and $66 \mathrm{~s}$ respectively. The decrease is evidence. In these two scenarios, the spillover in waiting areas 1 and 2 takes place frequently, resulting in ascent of right-turning and left-turning vehicle delays in East/West leg accordingly. Under this circumstance, the green time of phase $2-g_{2}-$ should be decreased, which can reduce the number of vehicles entering the waiting areas and accelerate the turns of right of way at the intersection. Meanwhile, it can also reduce the leftturning vehicle delay induced by spillover. The changing characteristic of optimal timing plan at HT intersection is different from that of traditional intersection. At traditional intersection, the cycle length would be increased to enlarge the intersection capacity.

Due to the decrease of cycle length, the delay of through vehicles in East/West leg is increased. Comparing with scenario $\mathrm{C} 1$, the increased percentages of E2 under scenarios C3 and C4 are not very significant, which are $15 \%$ and $22.1 \%$ respectively. This is because in East/West leg there are dedicated lanes for through vehicles. The movements of through vehicles are not affected by the spillover. However, the increased percentages of E1 under scenarios C3 and C4 are significant, which are 36 and $44.7 \%$ respectively. The reason for this is the arrival number of right-turning vehicles at each cycle exceeds the capacity of the waiting areas, which leads to the frequently spillover in waiting areas 1 and 2 . One right-turning vehicle needs about two signal cycles to cross the intersection.

The increase of E1 and E2 leads to the increase of E3. However, E3 does not change as drastically as E1. As the traffic demand and volume ratios in South and North legs are fixed, the ratio of right-turning vehicle volume in East/West leg to total vehicle volume of the intersection is relatively low. Thus, the significant increase of E1 does not lead to the same change of E3.

Table 4. The signal-timing plans and collected evaluation indexes $[s]$

\begin{tabular}{|c|c|c|c|c|c|c|}
\hline Scenario & $C$ & $g_{1}$ & $g_{2}$ & E1 & E2 & E3 \\
\hline $\mathrm{C} 1$ & 90 & 39 & 39 & 62.35 & 44.54 & 47.32 \\
\hline $\mathrm{C} 2$ & 88 & 38 & 38 & 69.18 & 48.61 & 52.25 \\
\hline $\mathrm{C} 3$ & 77 & 35 & 30 & 97.36 & 52.38 & 57.81 \\
\hline $\mathrm{C} 4$ & 65 & 28 & 27 & 112.83 & 57.16 & 68.37 \\
\hline
\end{tabular}

\section{Conclusions}

HT is an innovative traffic regulation scheme at road intersections to avoid the conflicts between right-turning vehicles and trams. This paper focused on the optimal signal-timing plan of the HT intersection, which is still an open question in the literature. Based on holistic analysis of the average vehicle delays in each signal phase, an optimization model is proposed for the optimal signal plan, with the aim of minimizing the average delay of all the vehicles in the intersection area. Due to the complexity of the objective function, a GA is proposed to solve the addressed problem.
A numerical test is built-up based on a realistic intersection with HT scheme in urban Melbourne. Field survey is conducted at this intersection in the morning peak and nonpeak periods. Based on the numerical test, it was shown that the optimal signal plan can significantly reduce the average vehicle delay, compared with the existing signal plan in both peak and nonpeak periods.

It should be noted that due to the limited space of the waiting area in the intersection, a spillback of the HT vehicles would commonly occur if the volume of right-turning vehicles is high. Such a spillback can inherently affect the movement of the vehicles. Hence, HT scheme is more suitable for the intersections with low right-turning volume. If the volume of right-turning vehicles is high, then some other traffic regulation schemes would be more suitable, including $\mathrm{U}$-turn and dedicated signal phase, etc.

\section{Acknowledgments}

This study is supported by the National Natural Science Foundation of China (No 61304198), the China Postdoctoral Science Foundation Funded project (No 2013M530159) and the China Postdoctoral Science Foundation Special Funded project (No 2014T70351).

\section{References}

Abukauskas, N.; Sivilevičius, H.; Puodžiukas, V.; Lingytė, I. 2013. Road safety improvement on at-grade intersections, The Baltic Journal of Road and Bridge Engineer 8(3): 212219. https://doi.org/10.3846/bjrbe.2013.27

Andrew O'Brien and Associates. 2000. Review of Hook Turns (Right Turn from Left). Research and Development Project 729. VicRoads, Melbourne, Australia.

Bugg, Z.; Rouphail, N. M.; Schroeder, B. 2013. Lane choice model for signalized intersections with an auxiliary through lane, Journal of Transportation Engineering 139(4): 371378. https://doi.org/10.1061/(ASCE)TE.1943-5436.0000513

Ceylan, H.; Bell, M. G. H. 2004. Traffic signal timing optimisation based on genetic algorithm approach, including drivers' routing, Transportation Research Part B: Methodological 38(4): 329-342.

https://doi.org/10.1016/S0191-2615(03)00015-8

Chen, P.; Liu, H.; Qi, H.-S.; Wang, F.-J. 2013. Analysis of delay variability at isolated signalized intersections, Journal of Zhejiang University SCIENCE A 14(10): 691-704. https://doi.org/10.1631/jzus.A1300208

Currie, G.; Reynolds, J. 2011. Managing trams and traffic at intersections with hook turns: safety and operational impacts, Transportation Research Record: Journal of the Transportation Research Board 2219: 10-19. https://doi.org/10.3141/2219-02

Currie, G.; Smith, P. 2006. Light rail and major activity center circulation systems: innovative design for safe and accessible light rail or tram stops suitable for streetcar-style conditions, Transportation Research Record: Journal of the Transportation Research Board 1955: 36-46. http://dx.doi.org/10.3141/1955-05

Highway Capacity Manual. 2000. Transportation Research Board. 1134 p. 
Hounsell, N. B.; Yap, Y. H. 2015. Hook turns as a solution to the right-turning traffic problem, Transportation Science 49(1): 1-12. https://doi.org/10.1287/trsc.2013.0482

Liu, Z.; Wang, S.; Meng, Q. 2014. Optimal joint distance and time toll for cordon-based congestion pricing, Transportation Research Part B: Methodological 69: 81-97. https://doi.org/10.1016/j.trb.2014.08.005

Liu, Z.; Yan, Y.; Qu, X.; Zhang, Y. 2013. Bus stop-skipping scheme with random travel time, Transportation Research Part C: Emerging Technologies 35: 46-56. https://doi.org/10.1016/j.trc.2013.06.004

Pai, C.-W.; Hsu, J.-J.; Chang, J.-L.; Kuo, M.-S. 2013. Motorcyclists violating hook-turn area at intersections in Taiwan: an observational study, Accident Analysis \& Prevention 59: 1-8. https://doi.org/10.1016/j.aap.2013.04.034

Park, B.; Messer, C.; Urbanik, T. 1999. Traffic signal optimization program for oversaturated conditions: genetic algorithm approach, Transportation Research Record: Journal of the Transportation Research Board 1683: 133-142. https://doi.org/10.3141/1683-17

Teklu, F.; Sumalee, A.; Watling, D. 2007. A genetic algorithm approach for optimizing traffic control signals considering routing, Computer-Aided Civil and Infrastructure Engineering 22(1): 31-43. https://doi.org/10.1111/j.1467-8667.2006.00468.x

Wang, S.; Meng, Q; Yang, H. 2013. Global optimization methods for the discrete network design problem, Transportation Research Part B: Methodological 50: 42-60. https://doi.org/10.1016/j.trb.2013.01.006

Yang, Z.; Liu P.; Tian, Z.; Wang, W. 2012. Evaluating the operational impact of left-turn waiting areas at signalized intersections in China, Transportation Research Record: Journal of the Transportation Research Board 2286: 12-20.

https://doi.org/10.3141/2286-02 